\title{
Estudo do comportamento azeotrópico da mistura etanol-água em colunas de destilação convencional a diferentes condições de pressão
}

Fecha de recepción: 11 de octubre de 2016

Fecha de aprobación: 20 de diciembre de 2016

Pp. 55-70

DOI: https://doi.org/10.21158/23823399.v4.n2.2016.1627

\author{
Jeffrey León-Pulido ${ }^{I}$ \\ Universidad EAN \\ jleonp@universidadean.edu.co \\ Maicon Ferreira ${ }^{2}$ \\ UNIVATES \\ eng.maiconf@gmail.com \\ lctorress@gmail.com
}

Cómo citar este artículo/ To reference this article/ Comment citer cet article/ Para citar este artigo: León-Pulido, J.; Ferreira, M. (2016). Estudo do comportamento azeotrópico da mistura etanol-água em colunas de destilação convencional a diferentes condições de pressão. Revista Ontare, 4(2), 55-70 .DOI: https://doi.org/10.21158/23823399.v4.n2.2016.1627

\section{Resumo}

A destilação é uma técnica comum para a separação de correntes líquidas com dois ou mais componentes e é uma das operações unitárias mais importantes na indústria química. Desse modo, as colunas de destilação necessitam uma grande demanda energética para realizar a separação dos componentes. Sendo assim, o estudo tem como objetivo realizar uma análise do primeiro parâmetro que deve se selecionar para a modelagem e simulação da coluna fazendo uso do simulador de processos Aspen Plus ${ }^{\circledR}$. Além disso, foram analisados três diferentes tipos de modelos termodinâmicos, os quais foram comparados com os dados experimentais observando seu comportamento com relação aos valores calculados com o simulador. Pela observação dos aspectos analisados, conclui-se que o modelo termodinâmico mais apropriado para utilizar na simulação de uma coluna de destilação para uma mistura binária azeotrópica de etanol-água é o modelo UNIFAC. Também pode-se concluir que a pressão de 0,1 atmosférica apresentou o melhor resultado.

\section{Palavras-chave}

Destilação, azeótropo, modelo termodinámico, simulação.

\footnotetext{
${ }^{1}$ Profesional en Ingeniería Química (UIS-Co), MSc. en Ingeniería Química y PhD (c) en Ingeniería Química (UNICAMP-Br). Director de organizaciones, operaciones, procesos industriales y académicos. Especialista en intensificación de procesos, desarrollo de procesos/productos y simulación numérica. ORCID: http://orcid.org/0000-0002-3603-286X

2 Estudiante del programa de Ingeniería mecânica, de la universidad UNIVATES de Brasil.
} 


\title{
Study of the azeotropic behavior of the ethanol-water mixture in columns of conventional distillation at different pressure conditions
}

\begin{abstract}
Distillation is a common technique for the separation of liquid streams with two or more components that requires a high demand of energy and is one of the most important unitary operations in the chemical industry. Therefore, this study aims at performing an analysis of the first parameter to be selected for modeling and simulation of the column using the Aspen Plus ${ }^{\circledR}$ process simulator. In addition, three different types of thermodynamic models were analyzed, which were compared with the experimental data, observing their behavior in relation to the values that were calculated with the simulator. By observing the analyzed aspects, it is concluded that the most appropriate thermodynamic model to be used in the simulation of a distillation column for an azeotropic binary ethanol-water mixture is the UNIFAC model. It can also be concluded that the atmospheric pressure of 0.1 shows a better result.
\end{abstract}

\section{Keywords}

Distillation, azeotrope, thermodynamic model, simulation.

\section{Étude du comportement azéotropique du mélange eau - éthanol dans des colonnes de distillation conventionnelle à pression variable}

\section{Résumé}

La distillation est une technique conventionnelle permettant de séparer des courants liquides de deux composants ou plus nécessitant une demande énergétique conséquente et qui constitue l'une des opérations unitaires les plus importantes de l'industrie chimique. Cette étude a comme objectif de réaliser une analyse du premier paramètre devant être sélectionné afin de simuler et modéliser la colonne de distillation grâce à l'utilisation du simulateur de processus Aspen Plus ${ }^{\circledR}$. Nous avons par ailleurs analysé trois différents types de modèles thermodynamiques qui ont été comparés aux données expérimentales pour observer leur comportement par rapport aux valeurs calculées avec le simulateur. L'observation des aspects analysés conclue que le modèle thermodynamique le plus approprié pour être utilisé lors d'une simulation de colonne de distillation de mélange binaire azéotropique eau - éthanol est le modèle UNIFAC. Une autre conclusion révèle en outre qu'une pression atmosphérique de 0,1 offre de meilleurs résultats.

\section{Mots clefs}

Distillation, azéotrope, modèle thermodynamique, simulation. 


\section{Estudio del comportamiento azeotrópico de la mezcla etanol-agua en columnas de destilación convencional a diferentes condiciones de presión}

\section{Resumen}

La destilación es una técnica común para la separación de corrientes líquidas con dos o más componentes que requiere una gran demanda energética y se constituye en una de las operaciones unitarias más importantes en la industria química. Siendo así, este estudio tiene por objetivo realizar un análisis del primer parámetro que debe seleccionarse para el modelado y simulación de la columna haciendo uso del simulador de procesos Aspen Plus ${ }^{\circledR}$. Además, se analizaron tres tipos diferentes de modelos termodinámicos, los cuales fueron comparados con los datos experimentales, observando su comportamiento con relación a los valores calculados con el simulador. Por la observación de los aspectos analizados, se concluye que el modelo termodinámico más apropiado para utilizar en la simulación de una columna de destilación para una mezcla binaria azeotrópica de etanol-agua, es el modelo UNIFAC. También se puede concluir que la presión atmosférica de 0,1 presenta un mejor resultado.

\section{Palabras-clave}

Destilación, azeótropo, modelo termodinámico, simulación. 


\title{
1. Introdução
}

\begin{abstract}
s colunas de destilação necessitam uma grande demanda energética A para realizar a separação dos componentes. De acordo com Engelien e Skogestad (2005), cerca de 3\% de toda a energia consumida no mundo está associada aos processos de destilação, sendo que $95 \%$ dessa energia é utilizada para os processos de separação de misturas. Em vista disso, os estudos sobre as colunas são de grande relevância.
\end{abstract}

A destilação é uma técnica comum para a separação de correntes líquidas com dois ou mais componentes e é uma das operações unitárias mais importantes na indústria química (Luyben, 1996). No processo de destilação ocorre uma separação dos componentes, essa separação acontece devido aos componentes terem diferentes pontos de ebulição, tornando um componente mais volátil e o outro mais pesado, sendo que o mais volátil evapora e é descarregado no topo da coluna, e o mais pesado tende a permanecer na parte inferior da coluna. Essa separação depende de vários parâmetros, tais como, pressão, temperatura, dimensionamento da coluna, entre outros. Logo, uma definição correta destes dados resultará em uma elevada eficiência do processo.

Sendo assim, esse estudo tem como objetivo realizar uma análise do primeiro parâmetro que deve se selecionar para simulação da coluna, na qual pode se verificar o máximo de pureza que pode ser extraído da mistura. Em seguida será feito o estudo do comportamento azeotrópico da mistura binária etanol-água, em colunas de destilação convencional a diferentes condições de pressão. Além disso, serão analisados três diferentes tipos de modelos termodinâmicos, comparando-os com os dados experimentais disponível no Dechema, (1990). Consequentemente, ajustando esse parâmetro estão reduzindo-se os custos de operação de forma significativa. 
A metodologia do trabalho é classificada como pesquisa quantitativa, pois, de acordo com Ramos et. al. (2003), pesquisa com abordagem quantitativa é tudo que pode ser mensurado em número, classificado e analisado.

\title{
2. Conceito de coluna de destilação
}

\begin{abstract}
destilação é um processo que se remonta às origens da civilização, Asendo um feito fácil de reconhecer, que ao aquecer uma mistura de álcool e água, a fase vapor é mais rica em álcool que em água (Pulido, 2008; Pulido, 2011a; Pulido, 2011b). Hoje em dia, industrialmente, apesar de novos métodos de separação estarem continuamente sendo explorados, a destilação foi e continua sendo o principal etapa de separação utilizada nas indústrias químicas, pois permite obter altas taxas de produtos, além de ampla faixa de aplicação (Moussa, 2001), sendo que, na grande maioria das indústrias as colunas de destilação apresentam esquemas de controle linear e geralmente correspondem a controles lineares tipo cascata (Rovaglio; Ranzi; Biardi; Faravelli, 1990). Desse modo, o desenho e controle de uma coluna de destilação é de grande importância, já que permite obter correntes de produto com a pureza requerida, ou seja, para a venda ou para utilização em outros processos químicos (Bequette, 1998).
\end{abstract}

Segundo estudos realizados por Sanchez e Cardona (2005), devido às propriedades físico-químicas das misturas etanol-água, é impossível retirar completamente a água a pressão atmosférica como consequência da formação de uma mistura azeotrópica que implica na obtenção de mistura com um máximo de 95,6\% em peso de etanol.

As colunas de destilação são compostas por um condensador no topo, o qual tem a função de esfriar o vapor, que é o componente mais volátil, e transformá-lo em líquido, podendo ser do tipo total, parcial- 
vapor e parcial-liquído-vapor. Também é composta por um refervedor na base que aquece o componente mais pesado, que está na fase líquida. Nesse caso o refervedor sempre mandará uma quantidade de líquido de volta à coluna, já que o condensador depende do modelo.

\subsection{Estudo dos modelos termodinâmicos}

Existem três tipos de colunas de destilação no software Aspen Plus V8.4, aplicadas para promover a separação da mistura binária etanol-água. A primeira e mais simples, chamada Shortcut distillation design using the Winn-Underwood (DSTWU), utiliza Gilliland method, sendo que tem como propósito determinar a razão de refluxo mínima, número mínimo de etapas, e quer razão de refluxo real ou número real de estágios. É utilizada para colunas com uma alimentação e duas correntes de produtos.

A segunda, conhecida como Shortcut distillation rating using (Distl), utiliza Edmister method, tendo como finalidade determinar a separação com base na razão de refluxo, número de estágios e razão destiladoalimentação, e também é utilizada para colunas com uma alimentação e duas correntes de produtos.

Por último, a coluna Rigorous fractionation (RadFrac), aqual tem um maior grau de complexidade e executa uma avaliação e cálculos rigorosos para colunas individuais, sendo utilizada para destilação ordinária, absorventes, strippers, destilação extrativa e azeotrópica, destilação de três fases, destilação reativa.

Desse forma, deve-se primeiro realizar uma simulação manipulando a coluna DSTWU, para determinar os fatores citados acima. Em seguida se emprega a coluna Distl para determinar os condições citadas acima também, e por último utiliza-se a coluna RadFrac que tem maior complexidade, porém determina todos os elementos, e ainda pode variar um elemento em função de outro para conseguir um melhor resultado. 


\subsection{Simulação de colunas de destilação}

Na etapa de simulação de uma coluna de destilação é necessário informar vários parâmetros ao simulador. Resumindo, são dados referentes das propriedades, dados de entrada e especificações referentes ao processo, sendo que cada um tem uma grande importância para o resultado final. Nesse estudo estamos analisando o primeiro parâmetro que devemos inserir no simulador, referente às propriedades, mais especificamente, ao componente, à pressão e ao modelo termodinâmico. Além disso, após ser validado o modelo termodinâmico, é analisada a pureza versus a pressão da coluna de destilação. Essa primeira análise é realizada através do software Aspen Plus V8.4, e comparado com os dados experimentais disponíveis no Dechema, (1990). A segunda análise é realizada apenas no software.

\section{Descrição do modelo termodinâmico}

O Non-random two-liquida model (NRTL) é um modelo de coeficiente de atividade que correlaciona os coeficientes $r_{i}$ de um composto $\chi_{i}$ de atividade, com as suas frações molares em fase líquida em questão. O conceito de NRTL baseia-se na hipótese de Wilson que diz que a concentração local em torno de uma molécula é diferente da concentração de grandes quantidades. Esta diferença é devida a uma diferença entre a energia de interaç̧ão da molécula central com as moléculas da sua espécie e que, com as moléculas de outro tipo $U_{i j}$. A diferença de energia também introduz uma não-aleatoriedade ao nível molecular local (Renon, H.; Prausnitz, 1968). 
A equação para o modelo NRTL é:

\section{Equação 1. Modelo NRTL.}

$$
\ln Y i=\frac{\sum_{j} x j \tau j i G j i}{\sum_{k} x k G k j}+\sum_{j} \frac{x j G i j}{\sum_{k} x k G k j}\left(\tau i j-\frac{\sum_{m} x m \tau m j G m j}{\sum_{k} x k G k j}\right)
$$

A Universal Functiona-lgroup Activity Coeffients (UNIFAC) é um sistema semi-empírico para a predição de atividade não-eletrólito em misturas não ideais. A UNIFAC utiliza os grupos funcionais presentes nas moléculas que compõem a mistura líquida para calcular os coeficientes de atividade. Ao utilizar as interacções para cada um dos grupos funcionais presentes nas moléculas, bem como alguns coeficientes binários de interacção, a atividade de cada uma das soluções pode ser calculada (Fredenslund; Russell; Prausnitz, 2005).

No modelo UNIFAC, existem três principais parâmetros necessários para determinar a atividade de cada molécula no sistema. Em primeiro lugar, a área $R$ e o volume de superfície $Q$ são obtidos a partir da área de Van der Waals de superfície e volumes. Estes parâmetros dependem exclusivamente dos grupos funcionais individuais sobre as moléculas do hospedeiro. Finalmente, há o parâmetro $\tau_{i j}$ de interação binário, o qual está relacionado com a energia $U_{i}$ de interação de pares moleculares.

A equação para o coeficiente de atividade original modelo UNIFAC líquido é constituído por um termo combinatório e residual:

Equação 2. Modelo UNIFAC.

$$
\begin{gathered}
\ln y=\ln y_{i}^{c}+\ln y_{i}^{r} \\
\ln y_{i}^{c}=\ln \left(\frac{\Phi_{i}}{\mathrm{x}_{\mathrm{i}}}\right)+\mathbf{1}-\frac{\Phi_{i}}{\mathrm{x}_{\mathrm{i}}}-\frac{\mathrm{Z}}{2}\left[\ln \frac{\Phi_{i}}{\theta_{i}}+1-\frac{\Phi_{i}}{\theta_{i}}\right]
\end{gathered}
$$


Estes dois modelos NRTL e UNIFAC são o foco deste estudo. Os modelos são analisados variando a pressão de operação, tendo como base uma mistura binária de etanol-água.

\subsection{Estudo do Azeótropo}

A destilação azeotrópica é um processo muito conhecido e difundido, tendo o seu início por volta dos anos 20 (Vasconcelos; Brito; Vasconcelos, 2010).

O termo azeótropo é usado para descrever o estado em que um líquido em ebulição produz um vapor com exatamente a mesma composição e, consequentemente, o líquido não muda de composição à medida que ele evapora (Smith; Ness; Abbott, 2000). E a separação por simples destilação torna-se impossível. Assim, uma das técnicas empregadas é a destilação azeotrópica, onde é adicionado um agente de arraste, com o objetivo de formar um novo azeótropo com um dos componentes inicialmente presentes na mistura (Ribeiro; Vasconcelos, 2004). Nesse estudo, a mistura tem potencial para formar ponto azeotrópico ao longo da coluna cercana a 0,81 em concentração molar aproximadamente. Então, nesse caso, o agente de arraste está presente na própria corrente de alimentação.

\section{Set da simulação}

Uom relação ao set da simulação, é utilizada a coluna RadFrac, pois é Os parâmetros referentes ao fluxo de entrada pode ser visualizado na tabela 1. Então, nessa sessão definem-se os parâmetros utilizados em block. Primeiramente é definido que o tipo de cálculo é de equilíbrio, o número de estágios é 14 , sendo que o primeiro estágio representa o condensador e o último estágio reproduz o refervedor. O condensador é tipo total, o refervedor é modelo Kettle, a fase é vapor-líquido e a alimentação da coluna é de sétimo estágio. Em seguida, é definido que a pressão de operação é do fundo até o topo da coluna. 


\subsection{Condições de alimentação e de operação da coluna}

Na tabela 1 são demonstrados os dados utilizados para a simulação da coluna de destilação. Cada dado foi criteriosamente definido, tentando se aproximar o máximo de um caso real, sendo que o número de estágios é descrito por McCabe e Thiele, (1925).

Tabela 1. Dados utilizados na simulação

\begin{tabular}{|l|l|}
\hline \multicolumn{2}{|c|}{ Dados utilizados na simulação } \\
\hline \multirow{2}{*}{ Alimentação (Mole frac) } & 0,5 água \\
\cline { 2 - 3 } & 0,5 etanol \\
\hline Modelo termodinâmico & UNIFAC \\
\hline Temperatura de entrada $\left({ }^{\circ} \mathrm{C}\right)$ & 65 \\
\hline Pressão de entrada $(\mathrm{atm})$ & 1 \\
\hline Pressão de operação da coluna $(\mathrm{atm})$ & 0,$1 ; 0,5 ; 1,0 ; 5,0$ \\
\hline Coeficiente de vazão total $(\mathrm{kmol} / \mathrm{h})$ & 100 \\
\hline Número de estágios & 14 \\
\hline Estágio de alimentação & 7 \\
\hline Tipo de condensador & Total \\
\hline Pressão do condensador $(\mathrm{atm})$ & 1 \\
\hline Pressão do refervedor (atm) & 1 \\
\hline Razão de refluxo & 1,03767672 \\
\hline Fração de destilado para a alimentação & 0,5 \\
\hline
\end{tabular}

Fonte. Elaboração própria.

Foram realizadas as etapas descritas nos tipos de modelos de destilação para definir os parâmetros. O modelo termodinâmico foi definido na análise binária das propriedades de mistura. Dessa maneira, será realizada a simulação da coluna. 


\section{Análise e resultados}

Testa sessão de resultados serão analisados os dados gerados através 1 da simulação da análise binária da mistura etanol-água e também da simulação da coluna de destilação, utilizando os dados de entradas citados na tabela.

Dessa forma, foram gerados diagramas $y=f(x)$ a pressão constante. Os diagramas são divididos em dois tipos de modelos termodinâmicos: NRTL e UNIFAC, e também é divido em três tipos de pressões: pressão baixa, pressão ambiente e pressão supercrítica, segundo Maul (1999), quando uma substância é elevada acima de seus pontos críticos de temperatura e pressão, ela passa para uma condição chamada de estado fluido supercrítico. Além disso, são comparados com os dados experimentais disponíveis no DECHEMA, 1990, tornando o estudo mais confiável. Após ter conhecimento do melhor modelo termodinâmico, são analisados os níveis de pureza versos pressão da simulação da coluna. Desse modo, ao final da análise, é possível determinar o modelo termodinâmico e a pressão que se deve utilizar para simulações da mistura estudada.

Primeiramente realizou-se a análise do melhor termodinâmico, comparando através de gráficos e desvio padrão os dados gerados pelo software Aspen Plus V8.4 e os dados experimentais. O figura 1 está estruturado da seguinte maneira: a linha contínua representa os dados gerados pelo software; a linha conhecida como marcador representa os dados experimentais, ambos para o modelo termodinâmico NRTL; e a linha tracejada representa a forma ideal de separação. Então, verificam-se que os dados gerados pelo software estão próximos aos dados gerados experimentalmente. Também apresenta um desvio padrão de 0,278102034 . 
Figura 1. Dados NRTL versos Experimentais.

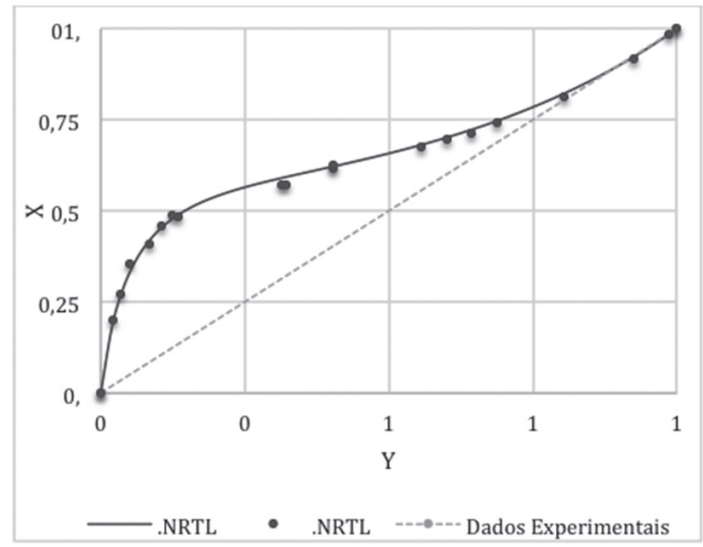

Fonte. Elaboração própria.

O figura 2 está estruturado da mesma forma que o gráfico acima, porém agora o modelo termodinâmico analisado é o UNIFAC. Também verifica-se que os dados gerados pelo software estão próximos aos dados gerados experimentalmente. Através desses dados, o resultado do desvio padrão é de 0,277753134 .

Figura 2. Dados UNIFAC versos Experimentais.

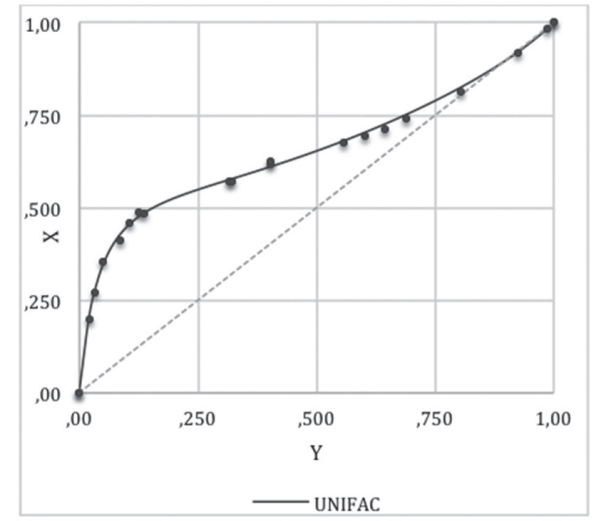

Fonte. Elaboração própria. 
Desse modo, podemos concluir que o modelo termodinâmico mais apropriado para utilizar na simulação da mistura binária etanolágua é o UNIFAC, pois apesar dos dois modelos apresentarem dados experimentais próximo aos do software, o modelo UNIFAC apresentou um menor desvio padrão.

Sendo assim, definido o modelo termodinâmico, será analisada a pureza versos pressão da coluna de destilação. Em consequência da simulação realizada utilizando os dados descritos no set de simulação, condições de alimentação e de operação da coluna. Na tabela 2 são demonstrados os resultados finais do estudo, nos quais observa-se que para 0,1 de pressão atmosférica a pureza do etanol no condensador é de 0,7928 e a pureza da água no refervedor é também de 0,7928 . Já para 0,5 de pressão atmosférica a pureza do etanol no condensador é de 0,7781 e a pureza da água no refervedor é de 0,7781 . Podemos verificar que há uma significativa diminuição na pureza conforme aumenta-se a pressão de operação da coluna. Isto pode ser visualizado para as outras pressões disponíveis na tabela 2.

Tabela 2. Dados de Pureza versos Pressão com modelo termodinâmico UNIFAC.

\begin{tabular}{|c|c|c|c|}
\hline \multicolumn{4}{|c|}{ Resultado Pureza versos Pressão } \\
\hline Componente & Condensador & Refervedor & Pressão \\
\hline WATER & 0,207163154 & 0,792836846 & \multirow{2}{*}{$0,1 \mathrm{~atm}$} \\
\hline ETHANOL & 0,79283685 & 0,20716315 & \\
\hline WATER & 0,221874918 & 0,778125082 & \multirow{2}{*}{$0,5 \mathrm{~atm}$} \\
\hline ETHANOL & 0,778125082 & 0,221874918 & \\
\hline WATER & 0,230096085 & 0,769903915 & \multirow{2}{*}{$1 \mathrm{~atm}$} \\
\hline ETHANOL & 0,769903918 & 0,230096082 & \\
\hline WATER & 0,25506132 & 0,74493868 & \multirow{2}{*}{$5 \mathrm{~atm}$} \\
\hline ETHANOL & 0,74493868 & 0,25506132 & \\
\hline
\end{tabular}

Fonte. Elaboração própria. 


\section{Conclusões}

Dela observação dos aspectos analisados, conclui-se que o modelo 1 termodinâmico mais apropriado para utilizar na simulação de uma coluna de destilação para uma mistura binária azeotrópica de etanol-água é o modelo UNIFAC, pois os dados experimentais desse modelo são mais convergentes com os dados gerados pelo software Aspen Plus V8.4, apresentando um desvio padrão menor do que o modelo NRTL.

Portanto, definido o modelo termodinâmico, foi realizada a simulação da coluna de destilação convencional, visando determinar a melhor pressão de operação da coluna para obter uma melhor pureza dos componentes no condensador e refervedor. Levando em conta o que foi observado, conclui-se que a pressão de 0,1 atmosférica apresentou o melhor resultado, pois teve um maior índice de pureza dos componentes. Além disso, percebe-se que quanto maior é a pressão de operação, menor é a pureza dos componentes da mistura. 


\section{Referências}

Bequette, B. (1998). Process Dynamics. Modeling, analysis and simulation. New Yersey, EEUU: Prentice Hall.

Engelien, H. K. and Skogestad, S. (2005), Minimum energy diagrams for multieffect distillation arrangements. AIChE Journal, 51, 1714-1725.

Fredenslund, A., Jones, R. L. and Prausnitz, J. M. (1975), Groupcontribution estimation of activity coefficients in nonideal liquid mixtures. AIChE Journal, 21, 1086-1099.

Gmehling, J.; Onken, U. (Eds.). (1990). Vapor-Liquid Equilibrium Data Collection, Chemistry Data Series. Frankfurt: DECHEMA.

Luyben, W. (2005). Process Modeling, Simulation and Control for Chemical Engineers. Mc-Graw Hill International Editions.

Maul, A. A. (1999).Fluidos supercríticos: situação atual e futuro da extração supercrítica. Biotecnologia ciência \& desenvolvimento, II(11), 42-46.

Mccabe, W. L.; Thiele, E. W. (1925).Graphical Design of Fractionating Columns. Industrial and Engineering Chemistry, 17, 605-611.

Moussa, S. L. (2001). Análise Termodinâmica de Colunas de Destilação Visando à Otimização Energética (Dissertação de Mestrado). Universidade Estadual de Campinas. Faculdade de Engenharia Química, Campinas, Brasil.

Pulido, L. J. (2008). Estudio de un nuevo concepto de columna de destilación: columna de destilación con integración interna de calor (HIDiC) (Trabajo de grado). Universidad Industrial de Santander. Escuela de Ingeniería Química. Bucaramanga, Colombia.

Pulido, J. L. (2011ª). Estudo de um novo conceito de coluna de destilação: coluna de destilação com integração interna de calor (CDIIC) (Dissertação de Mestrado). Universidad Estadual de Campinas, Brasil. 
Pulido, J. L.; Martinez, E L; Wolf, M. R.; Filho, R. M. (2011b). Heat transfer study of heat integrated distillation column (HIDiC) using simulation techniques. AIP - IAENG. Simulation. World Congress on Engineering \& Computer Science 2010 (WCECS 2010). San Francisco, USA, 742-747.

Sanchez, O.; Cardona, C. (2005). Producción biotecnológica de alcohol carburante ii: integración de procesos. INCI, 30,679-686.

Smith, J. M.; Ness, H. C. Van.; Abbott, M. M. (2000). Introdução à Termodinâmica da Engenharia Química. 5ed. Rio de Janeiro: Livros Técnicos e Científicos, cap 12, 390-395.

Ramos, P.; Ramos, M. M.; Busnello, S. J. (2003). Manual prático de metodologia da pesquisa: artigo, resenha, monografia, dissertação e tese. Blumenau: Acadêmica.

Renon, H.; Prausnitz, M. J. (1968). Local compositions in thermodynamic excess functions ofr liquid mixtures. AIChE Journal, 14(1), 135-144.

Ribeiro, O. M. S.; Vasconcelos, L .G. S. (2004). Simulação em regime estacionário de uma coluna de destilação azeotrópica de alta pureza. VIII Encontro Latino Americano de Iniciação Científica. Paraíba.

Rovaglio, M.; Ranzi, E.; Biardi, G.; Faravelli, T. (1990). Rigorous dynamics and feed- forward control design for distillation processes systems simulation and experimental results. Computers and Chemical Engineering, 14, 871-887.

Vasconcelos, S. L. A.; Brito, P. R.; Vasconcelos, S. G. L. (2010). Controle antecipatório aplicado a uma coluna de destilação azeotrópica não-convencional. VI Congresso Nacional de Engenharia Mecânica (CONEM). Campina Grande - Paraíba. 